Hou Yuan hang

Liang Xiao

Mu Xu yang

http://dx.doi.org/10.21278/brod69202

\title{
HULL LINES RELIABILITY-BASED OPTIMISATION DESIGN FOR MINIMUM EEDI
}

UDC 629.5.012: 629.5.016:519.6

Original scientific paper

\begin{abstract}
Summary
Pointing to marine greenhouse gas emissions, the EEDI (Energy Efficiency Design Index), a mandatory regulation, has become a significant factor in the design of ships' hull lines. EEDI is closely related to many design parameters of ships' hulls, which were conventionally set to be constant when involved in the design. However, it is often the case that considerable parameter fluctuations happen during actual navigation (such as travelling speed, draft, etc.), so it is more reasonable to state the important parameters as random variables. The reliability and quality level requirements of design results are also of concern. Accordingly, a reliability-based optimisation design (RBOD) method is introduced in this research. Furthermore, the design of experiments and reliability analyses via a Monte Carlo simulation and four reliability methods are employed to measure the sensitivity of the design variables and their reliability. Upon comparison with deterministic optimisation design (DOD) via adaptive simulated annealing (ASA), ROBD shows excellent adaptability and reliability in minimum EEDI ship hull lines' designs.
\end{abstract}

Key words: $\quad$ EEDI; Reliability-based optimisation; Ship hull lines; Sigma level

\section{Introduction}

The Energy Efficiency Design Index (EEDI) is a significant index for the energy efficiency of naval architecture, and it has been a mandatory regulation for all newly built ships since January 1, 2013 [1]. EEDI expresses the environmental cost, stated as the vessel's $\mathrm{CO}_{2}$ discharge, which is generated by the social benefit of each tonnage unit (quantity of shipments). The compulsory execution of EEDI accelerates the pace of energy saving and emission reductions in the shipping business, and higher requirements are proposed for the development of 'green' ships. It is well recognised that EEDI is closely related to ships' hull lines, which can greatly affect their sailing performance and other features. Therefore, along with the development of new ship hull lines, reducing EEDI and trends toward energy savings are becoming increasingly important. 
Currently, a number of internal and external parameters (such as resistance, travelling speed, draft, etc.) are normally set as constants in the design of hull lines (2014) [2,3]. However, the fluctuation of these parameters is unavoidable during actual navigation [4], which leads to the inevitable generation of errors if they are always considered as constant values. Although errors are small in most cases, during the optimisation design process, continuous iteration and mutual couplings may cause large deviations in the final results, and greater risks to quality come into view. Apparently, it is more reasonable to set such parameters as random uncertainty variables during the hull lines design process, so that their effect on target responses can also be considered.

In recent years, during ships' preliminary design stages, the influence of uncertain parameters has gradually come to be taken more seriously and subject to greater levels of research. Diez (2009) [5][6] took the lead in considering the influence of uncertain parameters on ship design: the principal dimensions of container ships were optimised using robust design (RD) and robust optimisation design (ROD). Afterwards, Diez's team (2011-2015) [710] introduced RD and ROD to ship hull simulation based design (SBD) systems, and a series of studies were conducted. Their design objective was developed from principal dimensions to hull lines, single uncertain parameters to multiple ones, and speed disturbances to wave responses, which reflected the development idea of 'from coarse to fine, from simple to complex'. Other works, such as that by Hannapel (2010) [11], introduced uncertain factors to constraint conditions, and the expectations and variances of objective functions were also considered. Papanikolaou and colleagues (2014) [12] made a review of methods on the uncertainties in the estimation of wave loads, and they studied ships' responses while in operation; in a later case, reliability assessment methods (FORM, SORM, MC) were used to account for the variations in ship and environmental parameters. The common characteristic of the above studies was that uncertain parameters were introduced into the fixed optimisation system as a priori knowledge, but the results' quality and reliability were not evaluated. Had they been, it would have led the design results to being of low quality with a high failure probability when applied to the further detailed designs.

From a statistical point of view, reliability-based optimisation design (RBOD) (2016) [13-15] considers the uncertainty of constraints as probabilistic at the beginning of the design process. By means of reliability analyses and quality optimisation, RBOD can yield the high quality results that strictly meet the requirements of performance, reliability and constraints. RBOD works by finding the flat area in a design space that minimises the output fluctuation caused by uncertain parameters and meets all quality requirements and probabilistic constraints.

In summary, with respect to previous approaches, Beyer (2007) lists the key factors of different definitions as follows [16]:

- Robust design (RD): Aiming at the worst case, attention is mainly on variances or standard deviation;

-Robust optimisation design (ROD): An optimisation process considering uncertainty in the objective or target function;

- Reliability-based optimisation design (RBOD): The attention is focused on the constraints of the design, which are treated as probabilistic inequalities and give a statistical feasible region.

While RD and ROD are mainly focused on expectations or variances of the objective function, RBOD concentrates on the probabilistic handling of constraints. Thus, RBOD can be a fine method for solving the way to approach quality requirements and probabilistic constraints. In this study, principal dimensions and ship form coefficients are combined to form the design space, and constraints are stated as hull moulded volume and resistance; thus, 
an optimisation model for minimum EEDI as the target is established. After the deterministic optimisation design (DOD) via adaptive simulated annealing (ASA), the design of experiments and reliability analyses via Monte Carlo simulation and several reliability methods are implemented to measure the sensitivity of design variables and the reliability of the optimisation results. Then, the RBOD is conducted for the final minimum EEDI hull line, which could well meet the quality requirements and probabilistic constraints.

\section{EEDI Calculation}

\subsection{EEDI formula and parameter}

EEDI is expressed by the ratio of $\mathrm{CO}_{2}$ emissions and quantity of shipments, and is related to the ship's fuel consumption, engine power, auxiliary power, energy-saving equipment, speed, tonnage and other factors [2]. Its calculation formula is shown as in Eq. (1):

$$
E E D I=\frac{E_{M E}+E_{A E}+E_{P T I}+E_{e f f}}{f_{i} \cdot \text { Capacity } \cdot v \cdot f_{w}}
$$

Where:

$$
\left\{\begin{array}{l}
E_{M E}=\left(\prod_{j=1}^{n} f_{j}\right)\left(\sum_{i=1}^{n M E} P_{M E(i)} \cdot C_{F M E(i)} \cdot S F C_{M E(i)}\right) \\
E_{A E}=P_{A E} \cdot C_{F A E} \cdot S F C_{A E} \\
E_{P T I}=\left(\prod_{j=1}^{n} f_{j} \sum_{i=1}^{n P T I} P_{P T I(i)}-\sum_{i=1}^{n e f f} f_{\text {eff }(i)} \cdot P_{A E \text { eff }(i)}\right) C_{F M E} \cdot S F C_{M E} \\
E_{\text {eff }}=-\left(\sum_{i=1}^{n e f f} f_{\text {eff }(i)} \cdot P_{\text {eff }(i)} \cdot C_{F M E} \cdot S F C_{M E}\right)
\end{array}\right.
$$

And where: $E_{M E}$ is $\mathrm{CO}_{2}$ discharge of main engine, and $E_{A E}$ is of auxiliary engine, $E_{P T I}$ is shaft belt device, $E_{\text {eff }}$ is energy-saving equipment. $C_{F}$ is carbon conversion coefficient, $v$ is travelling speed, $k n ; S F C$ is fuel consumption rate in $75 \%$ rated power, $g /(k W \cdot h) ;$ Capacity is deadweight tonnage, $t ; P$ is power for main or auxiliary engine, $k w ; f_{j}$ is modifying factor for ship special design, $f_{i}$ is modifying factor for ice strengthened ship, and is taken 1.0 for non ice strengthening; $f_{\text {eff }}$ is innovation factor, and is taken 1.0 for waste heat recovery unit, and for other energy or technology, it should be evaluated by classification society; $f_{w}$ is wind wave correction factor.

Parameters in this research are taken as in table. 1.

Tab.1 Parameter magnitude in EEDI formula

\begin{tabular}{l|l|l|l}
\hline Parameters & Magnitude & Parameters & Magnitude \\
\hline$S F C_{M E}$ & $190 \mathrm{~g} /(\mathrm{kWh})$ & $S F C_{A E}$ & $215 \mathrm{~g} /(\mathrm{kwh})$ \\
\hline$P_{M E}$ & $75 \% M C R_{M E}$ & $f_{i}, f_{j} 、 f_{w}$ & 1.0 \\
\hline \multirow{2}{*}{$P_{A E}$} & $0.025 M C R_{M E}+250\left(M C R_{M E} \geq 10000 \mathrm{~kW}\right)$ & $P_{A E e f f} P_{P T I} P_{\text {eff }}$ & 0 \\
\hline & $0.05 M C R_{M E}\left(M C R_{M E}<10000 \mathrm{~kW}\right)$ & 0 \\
\hline
\end{tabular}

Upon substitution of magnitudes in Tab.1 into Eq. (2), the simplified EEDI formula is obtained as Eq. (3): 


$$
E E D I=C_{F} \times \frac{142.5 M C R_{M E}+215\left(0.05 M C R_{M E}\right)}{\text { Capacity } \times v}=C_{F} \times \frac{0.5144 \times 153.25 R_{t}}{\text { Capacity } \times P C}
$$

Where: $M C R_{M E}$ is power rating of main engine, $(\mathrm{kW}) ; R_{t}$ is travelling resistance, $(\mathrm{kN})$; $P C$ is propulsive coefficient; Capacity is deadweight, $(t)$, got by Eq. (4):

$$
\left\{\begin{aligned}
\text { Capacity } & =\Delta-W_{t}=\rho \nabla-W_{h}-W_{o}-W_{m} \\
& =\rho \nabla-\left(C_{h}+C_{o}\right) L(B+D)-W_{m}
\end{aligned}\right.
$$

Where: $\Delta$ is displacement, $(t)$. $W_{t}$ is light weight, $(t)$, which is divided into hull weight $W_{h}$, outfit weight $W_{o}$ and electromechanical weight $W_{m}$. $W_{h}$ and $W_{o}$ can be estimated by the principal dimensions: waterline length $L(m)$, waterline width $B(m)$ and depth $D(m)$. Correspondingly, $C_{h}$ and $C_{o}$ are relevant coefficients, which are usually empirical valued. Electromechanical weight $W_{m}$ can be set as a fix value referred to parent type.

A large cargo ship is taken as the example in this study, detailed descriptions of which are shown later, according to the routine statistical data of this ship type [17], constant coefficients are defined as: $C_{h}=0.43, C_{o}=0.31, W_{m}=671(t)$ in this study.

\subsection{Travelling resistance $R_{t}$}

Numerical method based on the slender body theory such as Michell, is frequently-used in the ship resistance evaluation because of its simple assumptions and fast capabilities. However, such approach is considered out-dated and probably not accurate enough to capture the effect of small hull form modifications on the vessel resistance.

As a semi-empirical method for practical ship hull form, Holtrop method [18] with 334 towing test regressed data in ocean engineering basin in Netherlands, could get satisfactory resistance results at preliminary design stage. Because of the iterative computation in Holtrop, time cost of optimisation would increased substantially. Thus approximate model is necessary for the optimisation, which has strict requirement for the length of each step, and is also the typical and frequently-used way in ship simulation based design (SBD) process.

BP (Back Propagation) neural network is a kind of multilayer feed forward network with error back propagation algorithm [19], and becomes one of typical approximation technologies because of its excellent ability to approximate nonlinear function, Eq. (5) represents a three-layer BP neural network model which using tangent sigmoid as transfer function of neurons:

$$
\left.B P N N_{3}: O_{i}=\sum_{j=1}^{J} W_{i j} \tanh \left(\sum_{k=1}^{K} W_{j k} \tanh \sum_{n=1}^{N} W_{k n} \xi_{n}+b_{1 k}\right)+b_{2 j}\right)+b_{3 i}
$$

Where: $\xi_{n}$ is input variable, $O_{i}$ is output variable, $W_{k n}, W_{j k}, W_{i j}$ are the weights of the layers between neurons, $b_{1 k}, b_{2 j}, b_{3 i}$ are thresholds of neuron unit in each layer.

BP neural network approximate model, which is very sensitive to the internal parameters, needs mounts of simulation results as inputs, thus errors of output would appear due to some uncontrollable factors. Although this error or uncertainty has a small value in most cases, large deviation of the whole system could also be generated by continuous iterative computation. Therefore, considering the uncertainty of approximate model has an important significance.

\section{Deterministic optimisation}

\subsection{Design variables}

This study takes a large cargo ship as the example. In the optimisation design, design variables are often identified by principal dimensions, such as waterline length $L$, waterline 
width $B$, and the modification of the hull shape which can be represented by the original data points. Multiplied hull modification functions are as shown in Eqs. (6)-(7):

$$
\begin{gathered}
\left\{\begin{array}{l}
y_{f}(x, z)=y_{f_{0}}(x, z) \cdot \omega(x, z) \\
y_{a}(x, z)=y_{a 0}(x, z) \cdot \omega(x, z)
\end{array}\right. \\
\omega(x, z)=1-\sum_{m} \sum_{n} A_{m n} \sin \left[\pi\left(\frac{x-x_{0}}{x_{\max }-x_{0}}\right)^{m+2}\right] \cdot \sin \left[\pi\left(\frac{z_{0}-z}{z_{0}+T}\right)^{n+2}\right]
\end{gathered}
$$

Where: $y_{f(a)}(x, z)$ represents forward (or after) half of the hull points after changed, both in the mid ship-section of the interface; $\omega(x, z)$ is modification function of hull form; $A_{m n}$ is control factor for hull shape, in this research $m, n=1,2,3$.

\subsection{Constraints}

In order to ensure that the internal space of hull is not significantly reduced, and the travelling performance maintains acceptable, changing of molded volume and travelling resistance is taking as constraint condition:

$$
\left\{\begin{array}{c}
\nabla \geq \nabla_{0} \\
R_{t} \leq R_{t 0}
\end{array}\right.
$$

Where: $\nabla$ and $\nabla_{0}$ are optimal and initial hull form's molded volume, which can be calculated via "Simpson method" by the hull points; $R_{t 0}$ is initial hull form's resistance.

\subsection{Deterministic Optimisation Design (DOD) model and result}

After integrating the constraints into the optimisation objective, minimum EEDI optimisation model is established, as in shown in Tab.2:

Tab.2 Deterministic optimisation model for minimum EEDI hull lines

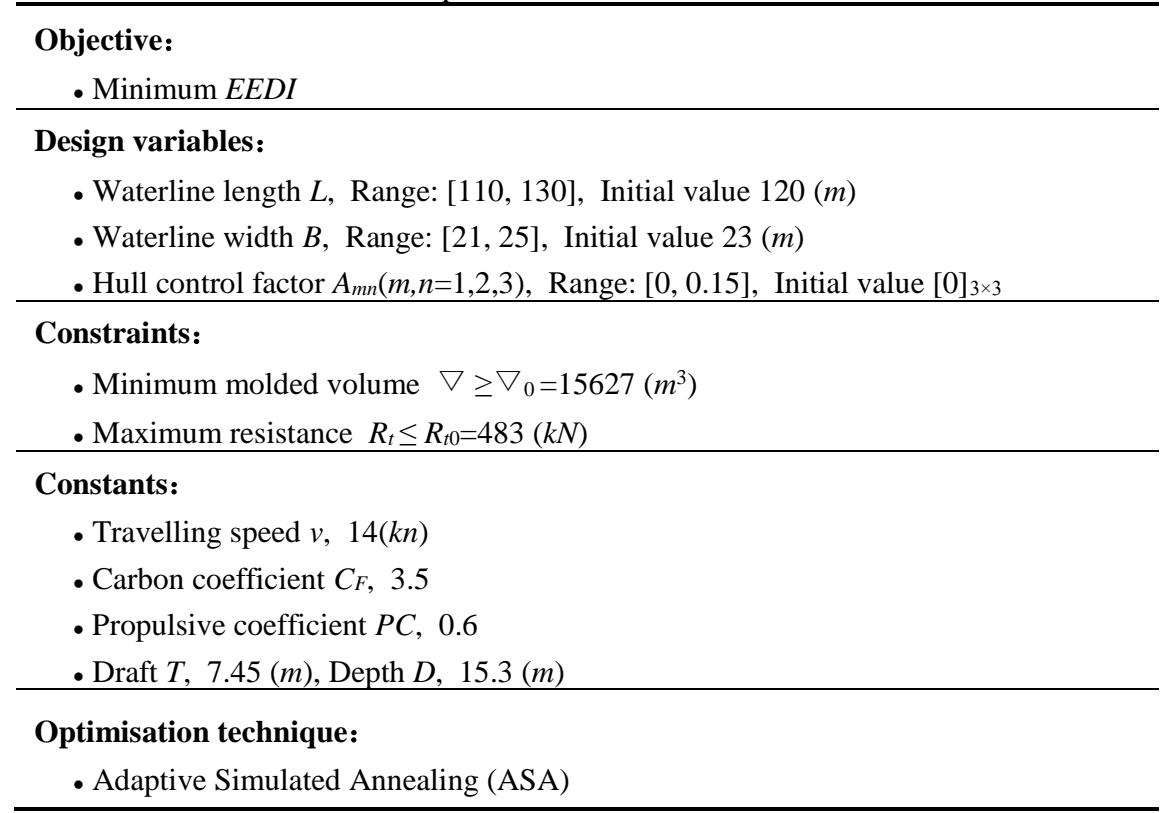

BP neural network, with its great ability to improve the computational efficiency, is adopted here to establish the approximate model of $R_{t}$. When training neural networks, a large number of experimental design points (sample points) should be distributed in the design space. The accuracy of neural network is directly affected by the number of and the distribution of sample points. Meanwhile, too much sample points cost a high computational cost. To compromise this contradiction, and to ensure the uniformity of sample distribution, 
uniform design method is employed here: uniform design table of 11 factors and 16 levels $U_{16}^{*}\left(16^{11}\right)$ is used and 16 sets of experimental program are generated. In order to scatter the sample points evenly, 50 sub-schemes are randomly generated in the range of input variables set by each experimental program, therefore, a total of 800 schemes were generated.

The parameters of BP neural network are chosen as: layer number is 3 , and the number of neurons in the input layer, the middle layer and the output layer are all $(12,6,1)$, convergence threshold is $1 \times 10^{-6}$. Adaptive learning rate method is used as the training method here, with an additional momentum factor, which improves speed and ensures that the network does not fall into local minima.

As an exploratory technique, Adaptive Simulated Annealing (ASA) [20] algorithm, which is very well suited for solving highly non-linear problems with short running analysis codes, when finding the global optimum is more important than a quick improvement of the design, is introduced here to do the optimisation.

The optimisation process is shown in Fig.1:

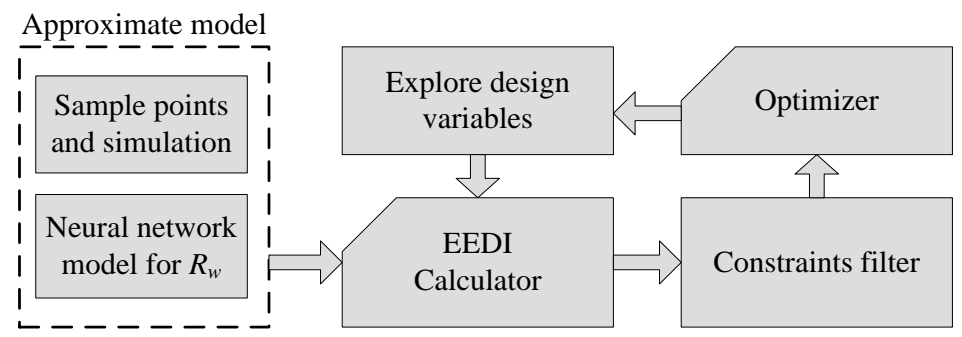

Fig.1 Deterministic optimisation process for minimum EEDI

The main internal parameters of ASA are set to: Max number of generated designs is 500 , relative rate of parameter annealing is 1.0 , and convergence epsilon is $1 \mathrm{e}-8$. After 520 iterations, optimal solution is obtained at the $506^{\text {th }}$ step. The result is shown in Tab.3, and the objective function values curve in optimisation process is shown in Fig.2.

Tab.3 Deterministic optimisation design (DOD) result

\begin{tabular}{c|c|c|c|c}
\hline \multirow{4}{*}{ Inputs } & Variables & Illustration & $\begin{array}{c}\text { Initial } \\
\text { scheme }\end{array}$ & DOD result (by ASA) \\
\cline { 2 - 5 } & $L(m)$ & Waterline length & 120.0 & 123.6 \\
\cline { 2 - 5 } & $B(m)$ & Waterline width & 23.0 & 24.5 \\
\hline \multirow{5}{*}{ Outputs } & $\begin{array}{c}A_{m n} \\
m, n=1,2,3)\end{array}$ & $\begin{array}{c}\text { Hull control } \\
\text { factors }\end{array}$ & {$[0] 3 \times 3$} & $\begin{array}{c}{[0.00180 .01320 .0890 .1020 .0330 .001} \\
0.0100 .00720 .0301]\end{array}$ \\
\cline { 2 - 5 } & $\nabla\left(m^{3}\right)$ & Molded volume & 15627 & 15690 \\
\cline { 2 - 5 } & $R_{t}(k N)$ & Resistance & 483 & 452 \\
\hline
\end{tabular}




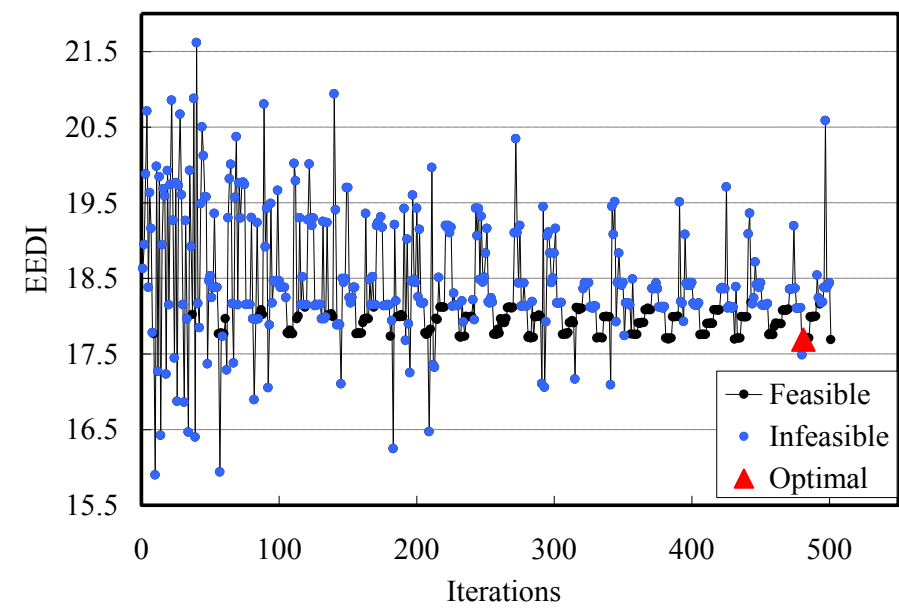

Fig.2 Objective function values curve in deterministic optimisation design process

\section{Sensitivity analysis}

In order to analyze the influence of variable changing on outputs, it's necessary to take the sensitivity analysis of design variables and important constants to the constraints and optimisation objective. The sensitivity analysis execution plan is shown in Fig.3.

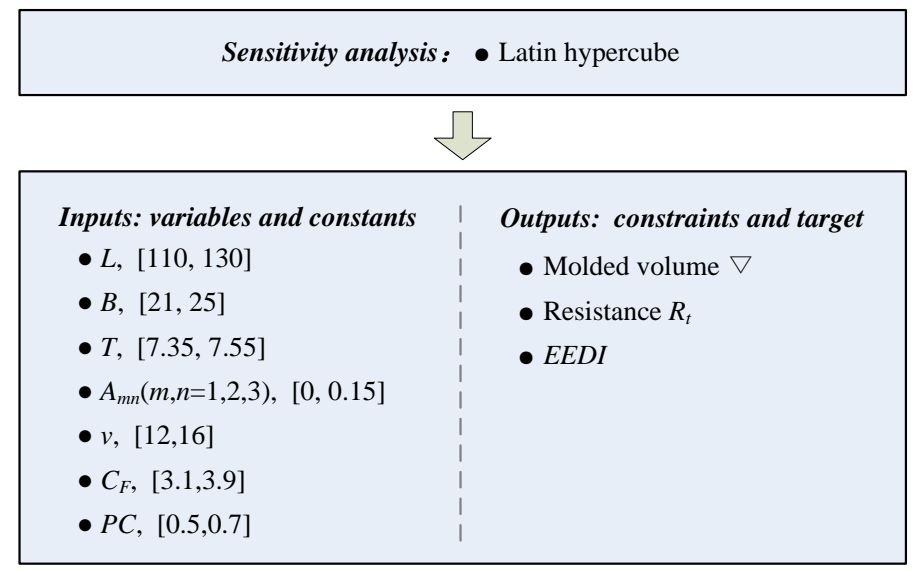

Fig.3 Sensitivity analysis execution plan

One experiments design technique is introduced here: Latin hypercube, because its engineer has total freedom in selecting the number of designs to run. 1500 points are generated for the Latin hypercube. Pareto contributions of inputs are shown in Fig.4, which can reflect the effect degree of each input on each output.

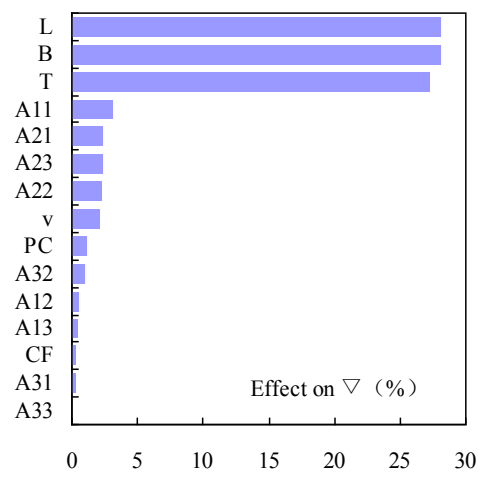

(a)

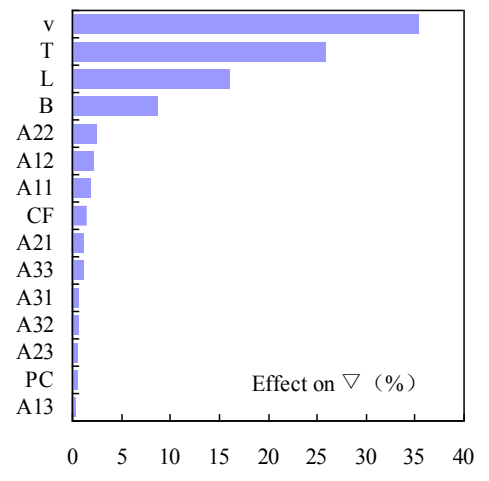

(b)

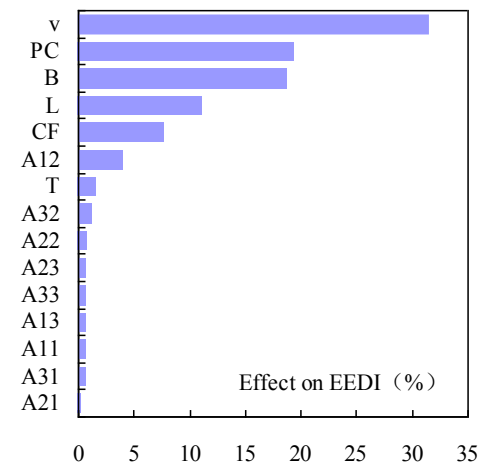

(c)

Fig.4 Pareto contribution of inputs to outputs 
As is shown in Fig.4, input factors such as $L, B, T, v, C_{F}, P C$ and their mutual operation customarily have a great effect on the three outputs. Nevertheless, effect of hull control factor $A_{m n}(m, n=1,2,3)$ is less important than others. In order to reduce the computational complexity, it's necessary to extrude the main factors and ignore the unimportant factors for the following reliability analysis and robustness design. Thus through sensitivity analysis, input factors: $L, B, T, v, C_{F}$, and $P C$ are taken into account as significant factors to the outputs response.

\section{Reliability analysis}

In the actual design process, input factors are often subject to interference and reflect the uncertainty, which is usually represented in the form of random variables. Because random disturbance of parameters is neglected in deterministic optimisation, the optimal solution is easy to be near or exceed the "edge position" of feasible region. Once disturbance occurs, deterministic optimal solution is likely to fall into the infeasible region, thus its reliability is hard to be guaranteed.

Reliability is the probability that the performance of result satisfies the constraint. Quality level is a comprehensive index to measure design quality, and two most typical quality levels are " $3 \sigma$ level" and " $6 \sigma$ level". The relationship between reliability and quality level is shown in Fig.5. For hull lines preliminary design, the $3 \sigma$ level of $99.73 \%$ reliability is sufficient.

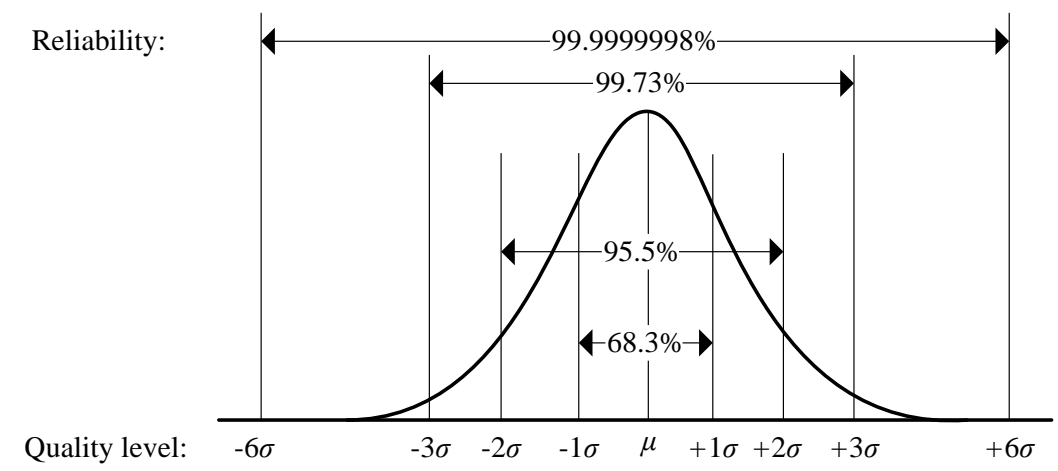

Fig.5 Quantization of reliability and quality level

To analysis the reliability and quality level of deterministic optimal result in Sec.2.3, Four typical reliability analysis methods are introduced here:

(1) Monte Carlo Simulation (MCS)

In MCS, the probability distribution of random variables is known. Through random sampling, the probability distribution of system response can be estimated and the contribution of each random variable to the response results can also be obtained. Illustration of MCS is as shown in Fig.6.
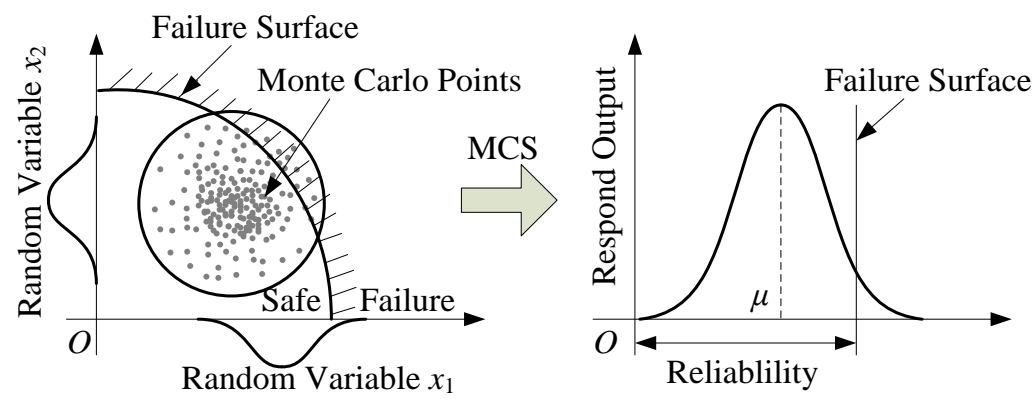

Fig.6 Illustration of Monte Carlo Simulation (MCS) 
There are two sampling techniques in MCS: simple random sampling and descriptive sampling. Compared to the former, descriptive sampling is a variance reduction technique aimed at reducing the variance of the statistical estimates derived from the population data. On the other hand, descriptive sampling can ensure the quality of statistical analysis with less sampling times and simulation, so it becomes a representative method and is used in this study.

\section{(2) Mean Value Reliability Method (MVRM)}

As a probabilistic method, MVRM utilizes the Taylor's series expansion of failure functions at mean values of the random variables. MVRM is the most efficient of the reliability analysis methods in terms of the number of function evaluations, or simulation program executions. However, unless the failure functions are linear or quadratic with normally distributed random variables, mean-value reliability index would lose accuracy. Illustration of MVRM is shown in Fig.7.

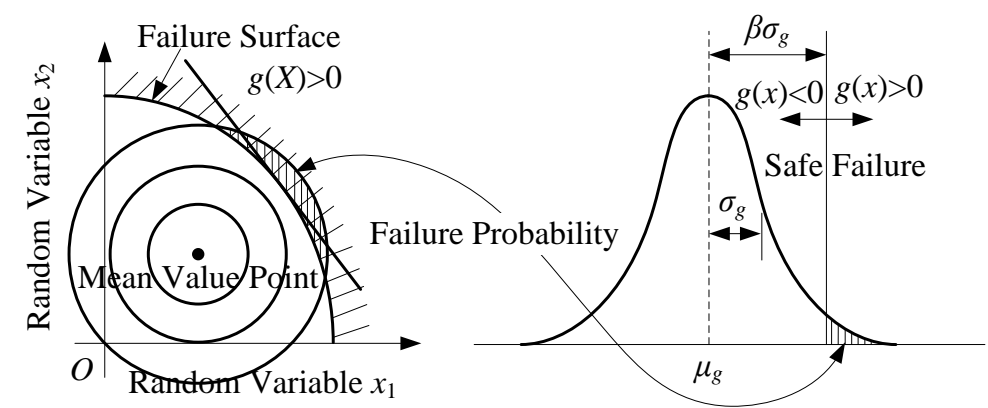

Fig.7 Illustration of Mean Value Reliability Method (MVRM)

(3) First Order Reliability Method (FORM)

FORM takes advantage of the desirable properties of the standard normal probability distribution, and reliability index is defined as the shortest distance from the origin of the standard normal space to a point on the failure surface. Mathematically, determining the reliability index is a minimization problem with one equality constraint.

Mean value and standard deviation of respond output of FORM is as Eq. (9)

$$
\left\{\begin{array}{l}
\mu_{y}=F\left(\mu_{x}\right) \\
\sigma_{y}=\sqrt{\sum_{i=1}^{m}\left(\frac{\partial F}{\partial x_{i}}\right)^{2}\left(\sigma x_{i}\right)^{2}}
\end{array}\right.
$$

Where: $x_{i}$ is random variable.

\section{(4) Second Order Reliability Method (SORM)}

When failure function for MPP (Probable Point Most) is nonlinear, SORM can get more accurate in the surface failure curvature of the approximate evaluation than FORM.

Mean value and standard deviation of respond output of SORM is as Eq. (10)

$$
\left\{\begin{array}{l}
\mu_{y}=F\left(\mu_{x}\right)+\frac{1}{2} \sum_{i=1}^{m} \frac{d^{2} F}{d x_{i}^{2}}\left(\sigma x_{i}\right)^{2} \\
\sigma_{y}=\sqrt{\sum_{i=1}^{m}\left(\frac{\partial F}{\partial x_{i}}\right)^{2}\left(\sigma x_{i}\right)^{2}+\frac{1}{2} \sum_{i}^{m} \sum_{j}^{m}\left(\frac{\partial^{2} F}{\partial x_{i} \partial x_{j}}\right)\left(\sigma x_{i}\right)^{2}\left(\sigma x_{j}\right)^{2}}
\end{array}\right.
$$

Problem model of FORM is shown in Fig.8. 

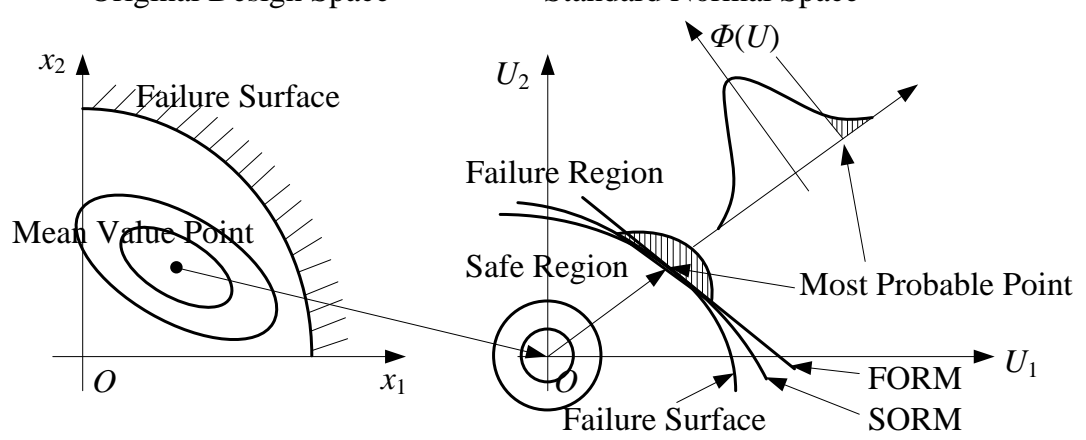

Fig.8 Illustration of FORM and SORM

Reliability analysis model of deterministic optimal result is shown in Tab. 4.

Tab.4 Reliability analysis model

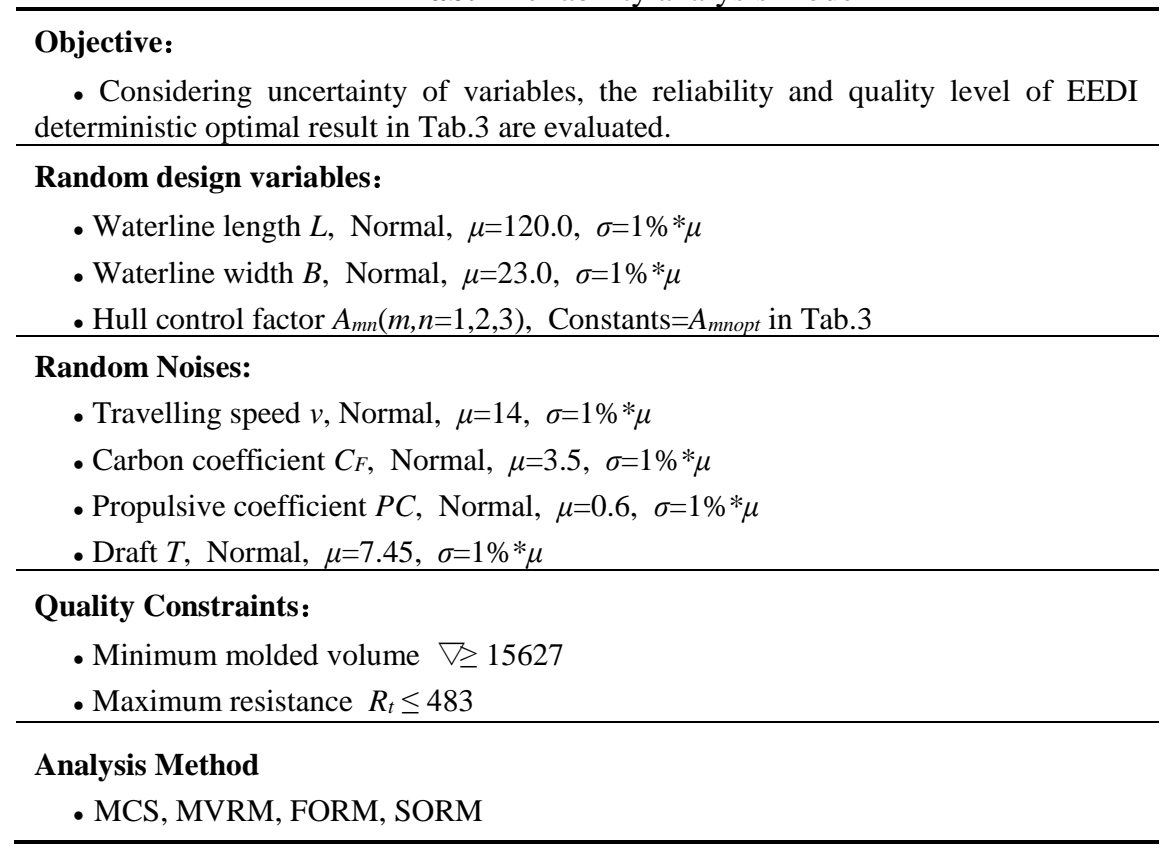

Because an accurate modeling of input distributions can be simply established or replaced from observed data, as is shown in Tab.4, normal distribution is assumed for all the random parameters (both the design variables and the noises) here. Furthermore, assessing probability density functions from observed data is outside the scopes of the present work and, therefore, no further addressed.

The optimisation objective EEDI and two constraints: molded volume and resistance are evaluated by the above four reliability analysis methods. Under the random disturbance of design variables and noises, their probability distributions are shown in Fig. 9. 

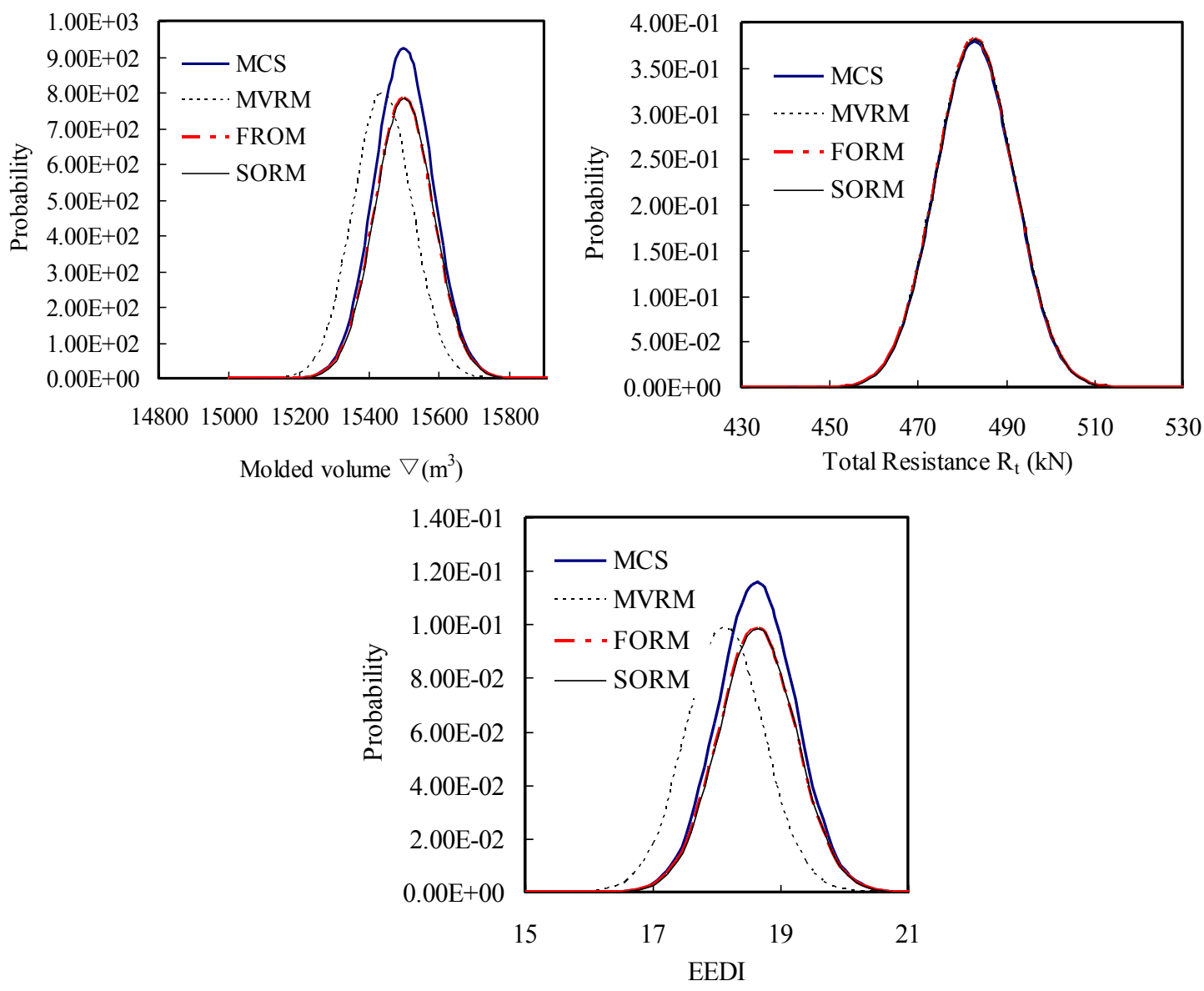

Fig.9 Probability distributions in reliability analysis

Reliability analysis results of molded volume, total resistance and EEDI are shown in Tab.5. Therefore, there are slight differences between the four reliability analysis results: expectations $\mu$ of MCS, FORM and SORM are basically the same, and the standard deviations $\sigma$ of MVRM, FORM and SORM are basically the same. Because MVRM needs the linear or quadratic failure functions, its analysis accuracy is less than others consequently.

Tab.5 Reliability analysis results

\begin{tabular}{|c|c|c|c|c|}
\hline & MCS & MVRM & FORM & SORM \\
\hline$\nabla$ & $\begin{array}{l}\mu=15584 ; \\
\sigma=0 ; \\
\text { Reliability: } 100 \% ; \\
\text { Quality Level: } 8\end{array}$ & $\begin{array}{l}\mu=15498 ; \\
\sigma=0 ; \\
\text { Reliability: } 98.34 \% ; \\
\text { Quality Level: } 2.39\end{array}$ & $\begin{array}{l}\mu=15584 ; \\
\sigma=231 ; \\
\text { Reliability: } 99.995 \% \\
\text { Quality Level: } 4.171\end{array}$ & $\begin{array}{l}\mu=15584 ; \\
\sigma=231 ; \\
\text { Reliability: } 99.995 \% \text {; } \\
\text { Quality Level: } 4.171 \\
\end{array}$ \\
\hline$R_{t}$ & $\begin{array}{l}\mu=489 ; \\
\sigma=5.73 ; \\
\text { Reliability: } 52.821 \% ; \\
\text { Quality Level: } 0.719\end{array}$ & $\begin{array}{l}\mu=489 ; \\
\sigma=6.71 ; \\
\text { Reliability: } 52.821 \% \text {; } \\
\text { Quality Level: } 0.719 \\
\end{array}$ & $\begin{array}{l}\mu=489 ; \\
\sigma=6.71 ; \\
\text { Reliability: } 52.756 \% ; \\
\text { Quality Level: } 0.718\end{array}$ & $\begin{array}{l}\mu=489 ; \\
\sigma=6.71 ; \\
\text { Reliability: } 52.821 \% \text {; } \\
\text { Quality Level: } 0.719 \\
\end{array}$ \\
\hline EEDI & $\begin{array}{l}\mu=18.98 \\
\sigma=1.005\end{array}$ & $\begin{array}{l}\mu=18.21, \\
\sigma=1.239\end{array}$ & $\begin{array}{l}\mu=18.98 \\
\sigma=1.239\end{array}$ & $\begin{array}{l}\mu=18.98 \\
\sigma=1.239\end{array}$ \\
\hline
\end{tabular}

Tab.5 shows that, the reliability of $R_{t}$ appears to be around $50 \%$, and quality levels are not reached 1 , that demonstrates the reliability of $R_{t}$ is extremely low. In order to improve its reliability and quality level, it's necessary to conduct the reliability optimisation design. 


\section{Reliability-based Optimisation Design}

Reliability-based optimisation design (RBOD) is to find a reliability result, which is far away from the failure surface. In the case of uncertain parameters, RBOD can effectively reduce infeasible probability, thus to improve the design result's reliability. Illustration of reliability optimisation is shown in Fig.10, and flow-process diagram of RBOD is shown in Fig.11.

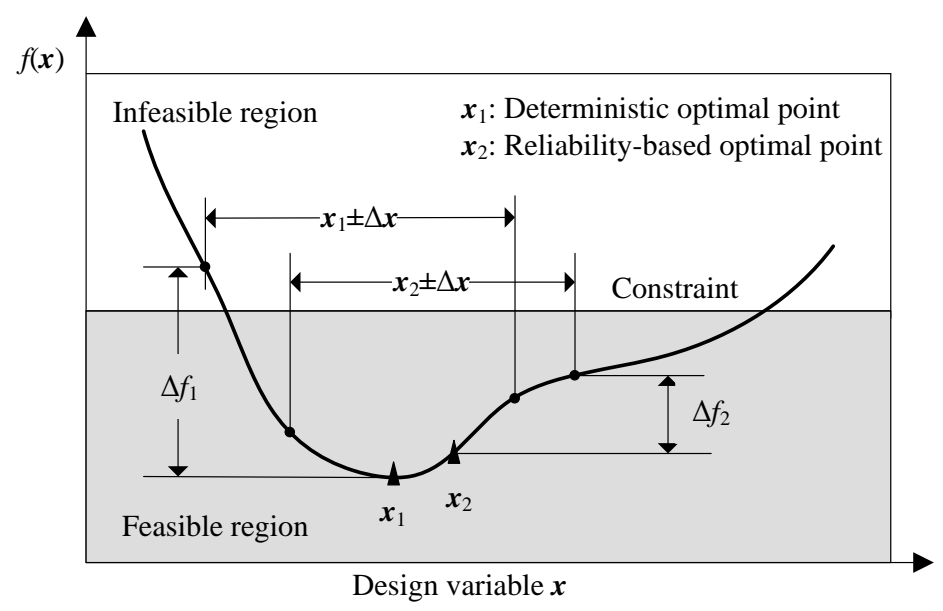

Fig.10 Illustration of reliability-based optimisation design

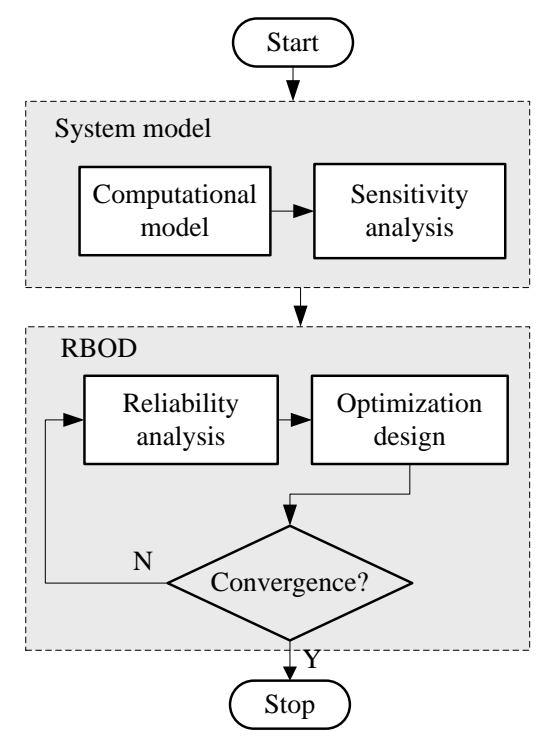

Fig.11 Flow-process diagram of reliability-based optimisation design (RBOD)

Mathematical model of reliability-based optimisation problem is expressed as in Eq.(11):

$$
\left\{\begin{array}{l}
\text { Minimize } F(\mu(X), \sigma(X)) \\
\text { Subject to } G(\mu(X), \sigma(X))<0 \\
X_{L S L}+\Delta X \leq X \leq X_{U S L}-\Delta X
\end{array}\right.
$$

Where: $X$ is random variables; $F$ and $G$ is target function and constraints function of RBOD, which are defined by expectation $\mu$ and standard deviation $\sigma ; \pm \Delta X$ is the fluctuation region of $X ; X_{L S L}$ and $X_{U S L}$ are lower bound and upper bound of design variables.

Target function F can be decomposed into two parts: "Mean on target" and "Minimize variation": 


$$
F=\sum_{i=1}^{l}\left[\frac{\omega_{1 i}}{S_{1 i}}\left(\mu_{i}-M_{i}\right)^{2}+\frac{\omega_{2 i}}{S_{2 i}} \sigma_{i}^{2}\right]
$$

Where: $i$ is component index of performance parameter set; $M$ is expected average performance target; $\omega_{1}$ and $\omega_{2}$ are weights of expectation $\mu$ and standard deviation $\sigma, S_{1}$ and $S_{2}$ are normalization coefficients of $\mu$ and $\sigma$.

Mathematical constraints $G$ in Eq.(14) can be converted to quality constraints $G_{q}$ which are expressed as "Sigma level", that can ensure the results quality in a specific sigma level. Quality constraints include: expectation $\mu$, standard deviation $\sigma$ and sigma level number $n$ :

$$
\left\{\begin{array}{l}
G_{q}=\mu+n \sigma \leq \text { Upper Limit } \\
G_{q}=\mu-n \sigma \geq \text { Lower Limit }
\end{array}\right.
$$

In Eq.(12), sigma level number $n$ represents strict level of result quality requirement. When $n= \pm 3$, namely " $3 \sigma$ Design", which reliability is $99.73 \%$ according to Fig. 5 , and is usually considered as the acceptable result quality. When $n= \pm 6$, that is " $6 \sigma$ Design", which reliability is $99.9999998 \%$, and can effectively reduce the per-million failure probability of results. As its huge time cost and high non- convergence risk, " $6 \sigma$ Design" is not used here. The quality level and reliability of " $3 \sigma$ Design" are enough for hull lines design in the ship preliminary design stage, and is adopted in this paper for the minimum EEDI hull lines RBOD.

Considering the uncertainty of variables, deterministic optimisation result in Sec. 2.3 is taken as the initial optimisation scheme. Aiming at finding the minimum $\mu$ and $\sigma$ of EEDI, and also meeting the $3 \sigma$ quality level of constraints, RBOD is conducting as follows. As its excellent accuracy evaluation performance, Second Order Reliability Method (SORM) is taken as the reliability analysis method. RBOD model of minimum EEDI hull lines is shown in Tab. 6.

Tab.6 The minimum EEDI hull lines RBOD model

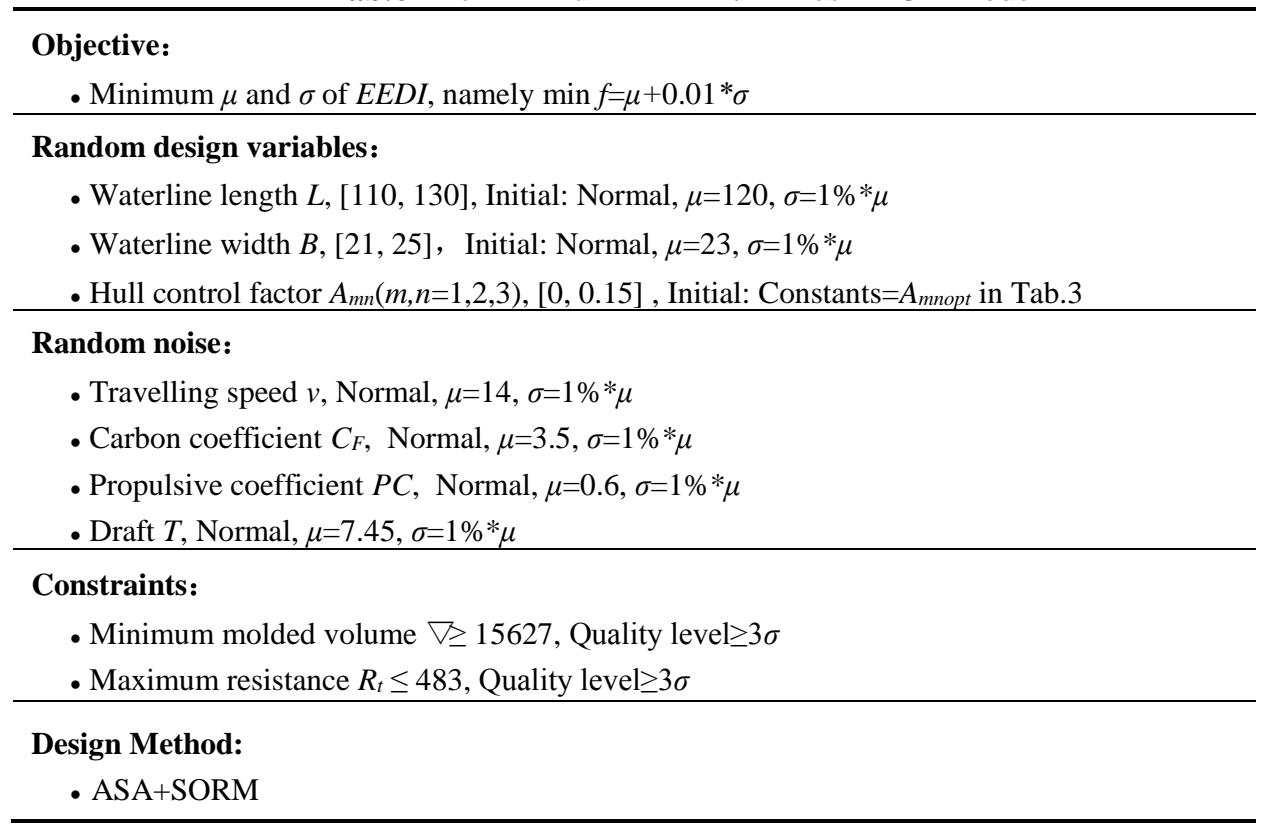

After conducting of RBOD, comparison of deterministic optimisation result and RBOD result is shown in Tab.7. As an important parameter in hull form design, $L_{C B}$ (length between floating center and after-perpendicular) is given to indicate the hull longitudinal deformation, as in Tab.7. 
As is shown, the optimisation objective EEDI of RBOD is slightly worse than the DOD result, but the two constraints: molded volume and total resistance are more far away form failure surface. Therefore, RBOD result has stronger immunity for the disturbance of uncertain factors.

Tab.7 Results comparison of two optimal design method

\begin{tabular}{|c|c|c|c|c|c|c|}
\hline & Variables & $\begin{array}{c}\text { Initial } \\
\text { scheme }\end{array}$ & \multicolumn{2}{|r|}{ DOD result } & \multicolumn{2}{|c|}{ RBOD result } \\
\hline \multirow{4}{*}{ Inputs } & $L(m)$ & 120.0 & & 123.6 & \multicolumn{2}{|r|}{121.9} \\
\hline & $B(m)$ & 23.0 & \multicolumn{2}{|r|}{24.5} & \multicolumn{2}{|r|}{23.3} \\
\hline & $\begin{array}{c}A_{m n} \\
(m, n=1,2,3)\end{array}$ & {$[0]_{3 \times 3}$} & \multicolumn{2}{|c|}{$\left[\begin{array}{ccccc}0.0018 & 0.0132 & 0.089 & 0.102 & 0.033 \\
0.001 & 0.010 & 0.0072 & 0.0301\end{array}\right]$} & \multicolumn{2}{|c|}{$\begin{array}{c}{\left[\begin{array}{lllll}0.0016 & 0.0101 & 0.103 & 0.102 & 0.045 \\
0.003 & 0.098 & 0.004 & 0.0217\end{array}\right]}\end{array}$} \\
\hline & $L_{C B}(m)$ & 55.32 & \multicolumn{2}{|r|}{54.01} & \multicolumn{2}{|r|}{54.90} \\
\hline \multirow{3}{*}{ Outputs } & $\nabla\left(m^{3}\right)$ & 15627 & 15690 & $\begin{array}{l}\text { Reliability: } 99.995 \% \text {; } \\
\text { Quality Level: } \mathbf{4 . 1 7 1}\end{array}$ & 15631 & $\begin{array}{l}\text { Reliability: } 99.999 \% \text {; } \\
\text { Quality Level:4.683 }\end{array}$ \\
\hline & $R_{t}(k N)$ & 483 & 452.3 & $\begin{array}{l}\text { Reliability: 52.821\%; } \\
\text { Quality Level: } \mathbf{0 . 7 1 9}\end{array}$ & 467.8 & $\begin{array}{l}\text { Reliability: } 99.833 \% \text {; } \\
\text { Quality Level: } \mathbf{3 . 5 9 0} \\
\end{array}$ \\
\hline & EEDI & 18.63 & & 17.69 & \multicolumn{2}{|r|}{18.16} \\
\hline
\end{tabular}

Under the normal disturbance of design variables and noises, probability distributions and quality levels comparison of DOD and RBOD are as shown in Fig. 12 and Fig.13, which are analysis by SORM.
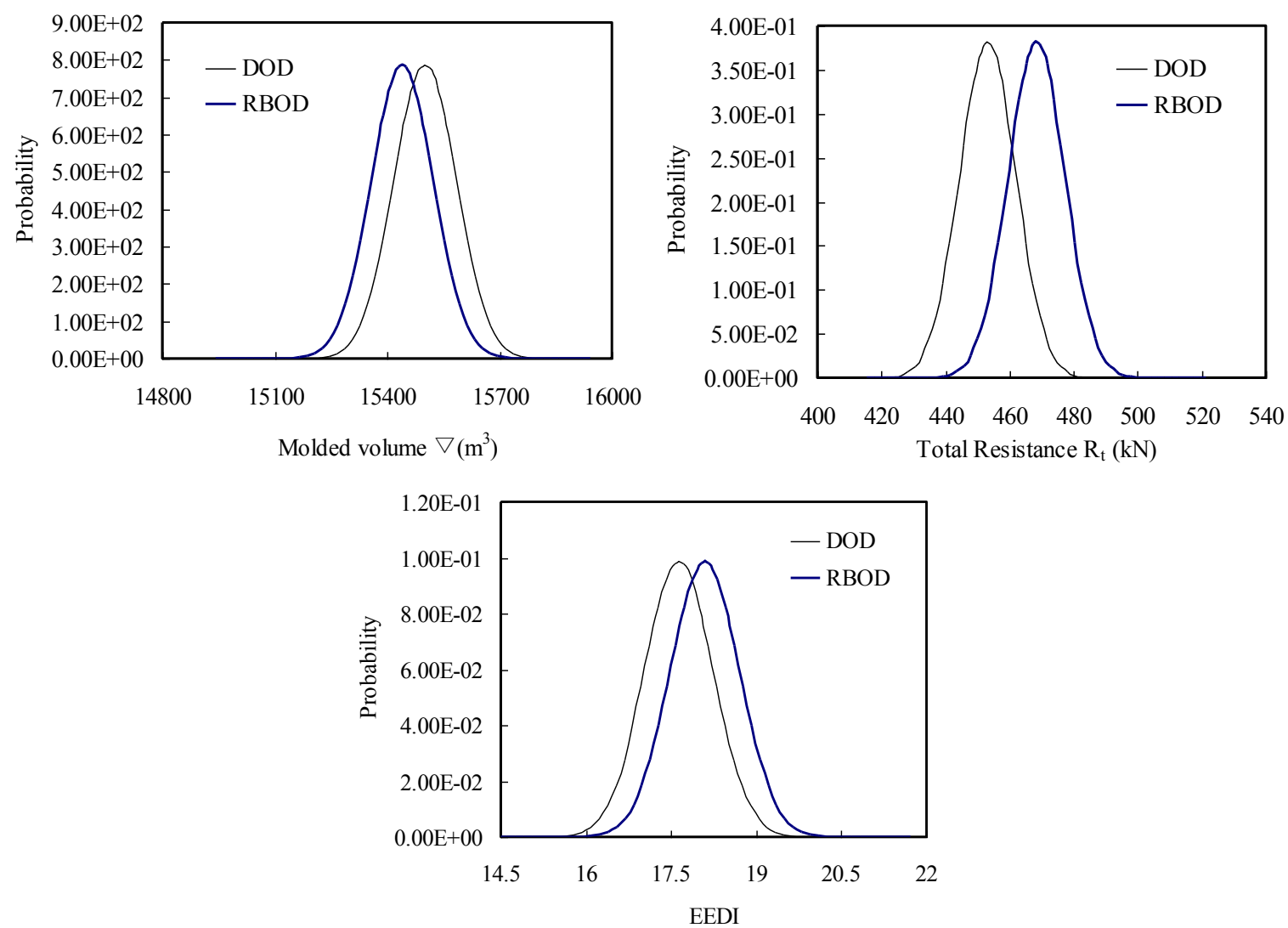

Fig.12 Probability distributions comparison of DOD and RBOD 


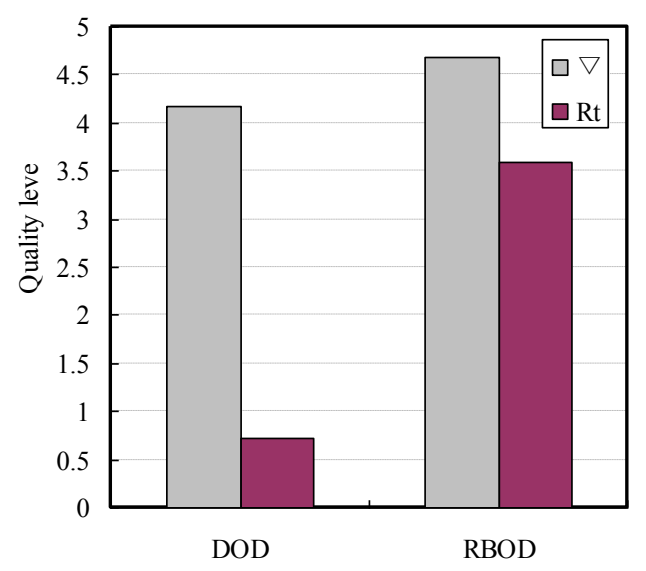

Fig.13 Quality levels of constraints comparison of DOD and RBOD

For hull lines comparison, section lines of the initial scheme, DOD result and RBOD result are showed in Fig.14. Apparently, the DOD result has the greatest shape changing (especially at the parallel middle body) and RBOD' change is comparatively placed in the middle, and bulbous bow of RBOD has some greater improvements. In conclusion, the RBOD method introduced in this paper can get a more reliability hull lines result and without great changing of the conventional DOD result.

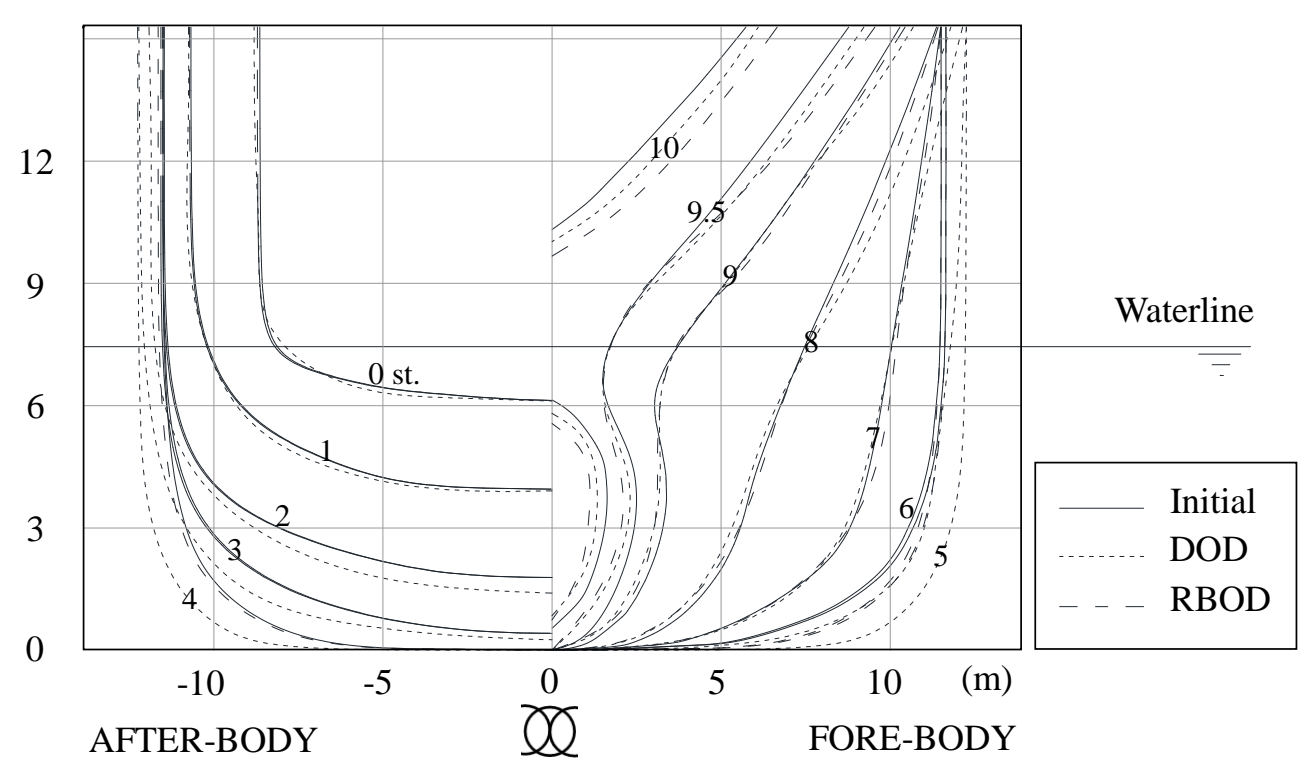

Fig.14 Section lines comparison

\section{Conclusion}

This paper considered the problems of hull lines design for the minimum EEDI in consideration of the uncertain parameters. Based on reliability-based optimisation design technology, a 3-sigma design target for quality level was conducted, which had more reliability for the actual environment disturbance. This paper researched theoretically the reliability analysis and optimisation method, and some critical assessments could be got:

(1) Both the reduction of resistance and increase of molded volume could cause deduction of EEDI, as is the goal of this paper. But the two are very contradictory, thus in finally results, as in Tab.7, the changes of molded volume are insignificant, while changes of resistance are greater. Introduction of some designer preferences to the optimisation process may be a better approach to reconcile this contradiction. 
(2) As an operation indicator, parameters of EEDI do not change during operation and EEDI is actually fixed when shipping. In order to get the best adaptability of EEDI, in the hull lines design stage, which has the important effect on EEDI, designers need to consider a variety of marine environmental uncertainty factors, not limited to this research.

(3) RBOD method can get a more reliability hull lines and without great changing of the conventional DOD result and initial, thus the reliability-based ship hull design has the feasibility and effectiveness. Further work could conduct from more reliability constraints and higher quality level for hull lines design and may other aspects of naval architecture.

\section{Acknowledgement}

This work is supported by the National Natural Science Foundation of China (Grant No. 51609030), and the Fundamental Research Funds for the Central Universities (No. 3132016358, No.3132016339, No.3132017017)

\section{REFERENCES}

[1] IMO-MEPC. 2014 Guidelines on the method of calculation of the attained energy efficiency design index(EEDI) for new ships. Resolution MEPC. 245(66), Adopted on 4 April 2014.

[2] Zou Y. Research on optimisation method for high performance vessel based on EEDI energy efficiency design index[D]. Dalian: Dalian Maritime University, 2014. 5-15.

[3] Cheng H R, Liu X D, Feng B W. Study on multidisciplinary optimisation method for hull forms design[J]. Shipbuilding of China, 2014, 55(1): 76-82.

[4] Ding J. Uncertainty analysis of ship velocity measurement in sea trial considering ship velocity algorithm[J]. Shipbuilding of China, 2007, 48(2): 144-148.

[5] Diez, M., Peri D. Global optimisation algorithms for robust optimisation in naval design[C]. In: 8th International Conference on Computer Applications and Information Technology in the Maritime Industries, COMPIT 2009.

[6] Diez, M., Peri D. Robust optimisation for ship conceptual design[J]. Ocean Engineering, 2010, 37: 966-977. https://doi.org/10.1016/j.oceaneng.2010.03.010.

[7] Wei H, Diez M., Zou Z J, etc. URANS study of Delft catamaran total/added resistance, motions and slamming loads in head sea including irregular wave and uncertainty quantification for variable regular wave and geometry[J]. Ocean Engineering, 2013, 74: 189-217. https://doi.org/10.1016/j.oceaneng.2013.06.020.

[8] Diez, M., Wei H, Campana E F. Uncertainty quantification of Delft catamaran resistance, sinkage and trim for variable Froude number and geometry using metamodels, quadrature and Karhunen-Loeve expansion[J]. J Mar Sci Technol, 2014,19: 143-169. https://doi.org/10.1007/s00773-013-0235-0.

[9] Leotardi C, Campana E F, Diez M. On the use of uncertainty quantification in variable-accuracy simulationbased multidisciplinary optimisation[C]. In: 3rd Workshop on Uncertainty Quantification in Computational Fluid Dynamics, 2015.

[10] Xi C, Diez M, Manivannan K, etc. High-fidelity global optimisation of shape design by dimensionality reduction, metamodels and deterministic particle swarm[J]. Engineering Optimisation, 2015, 47(4): 473-494. https://doi.org/10.1080/0305215X.2014.895340.

[11] Shari H, Nickolas V. Introducing uncertainty in multidiscipline ship design[J]. Naval Engineers Journal, 2010, 122(2): 41-52. https://doi.org/10.1111/j.1559-3584.2010.00267.x.

[12] Papanikolaou A, Alfred Mohammed, E., Hirdaris, S.. Stochastic uncertainty modelling for ship design loads and operational guidance[J]. Ocean Engineering, 2014, 86:47-57. https://doi.org/10.1016/j.oceaneng.2014.01.014.

[13] Li F Y, Rong J H, Hu L, etc. Reliability-based optimisation design of structure subjected to vehicle frontal impact based on probability-convex hybrid model[J]. Journal of Vibration and Shock, 2016.

[14] Dahoo P R, Pougnet P, Hami A E. Reliability- based Design Optimisation[M]// Nanometer- Scale Defect Detection Using Polarized Light. John Wiley \& Sons, Inc. 2016:25-45. 
[15] Baabbad M A, Nikolaidis E, Kapania R K. New Approach for System Reliability-Based Design Optimisation[J]. Aiaa Journal, 2015, 129(44):1087-1096.

[16] Beyer H G, Sendhofff B. Robust optimisation- a comprehensive survey[J]. Computational Methods in Applied Mechanics and Engineering, 2017:196.

[17] Cheng B, Pan W W. Ship design principle[M]. Shanghai: Shanghai JiaoTong University Press, 2007. 43-45.

[18] Holtrop J, Mennen GGJ. An approximate power prediction method[J]. International ship build progress, 1982, 29(335):166-170.

[19] Li Q H, Liu D., 2015. Aluminum Plate Surface Defects Classification Based on the BP Neural Network[J]. Applied Mechanics \& Materials, 734:543-547. https://doi.org/10.4028/www.scientific.net/AMM.734.543.

[20] Junior H A E O, Ingber L, Petraglia A, et al. Adaptive Simulated Annealing[J]. Intelligent Systems Reference Library, 2012, 35(5):33-62. https://doi.org/10.1007/978-3-642-27479-4_4.

Submitted: 13.06.2017. Hou Yuan hang (Family name: Hou, Given name: Yuan hang) Liang Xiao (Family name: Liang, Given name: Xiao)

Accepted: $12.12 .2017 . . \quad$ Mu Xu yang (Family name: Mu, Given name: Xu yang)

Email: houyuanhang6@163.com, Tel: +86 13940859887

Naval Architecture and Ocean Engineering College, Dalian Maritime University Linghai Road No. 1, High tech Zone, Dalian City, Liaoning Province, China, 116026 